\title{
CONSERVATION PRIORITIZATION OF HABITATS AND FOREST COMMUNITIES IN THE LAHAUL VALLEY OF PROPOSED COLD DESERT BIOSPHERE RESERVE, NORTH WESTERN HIMALAYA, INDIA
}

\author{
SINGH, A. ${ }^{1}-$ SAMANT, S.S. ${ }^{2 *}$ \\ ${ }^{I}$ G.B. Pant Institute of Himalayan Environment and Development Himachal Unit; \\ Mohal - Kullu - 175 126, Himachal Pradesh, India \\ ${ }^{2}$ Himachal Unit \& Theme Head, Biodiversity Conservation and Management, G.B. Pant Institute of \\ Himalayan Environment and Development Himachal Unit; Mohal - Kullu - 175 126, Himachal \\ Pradesh, India (phone: 01902-225329, ext. 21; fax: 01902-226347) \\ *Corresponding author \\ e-mail: Samantss2@rediffmail.com
}

(Received $12^{\text {th }}$ June 2008 ; accepted $25^{\text {th }}$ January 2010)

\begin{abstract}
The rapid loss of biodiversity due to habitat degradation and over exploitation has necessitated the conservation prioritization of habitats, species and communities for conservation. The prioritization of habitats and communities through qualitative and quantitative assessment of vegetation is prerequisite for initiating any conservation and management programme. Therefore, an attempt has been made to; (i) identify the habitats and communities; (ii) evaluate habitats and communities for species richness, native, endemic, economically important and threatened species; (iii) prioritize habitats and communities for conservation. Fifteen (15) habitats and fourteen forest (14) communities distributed between 2490-4000m were recorded. Overall, $35.71 \%$ communities were broad-leaved, $57.14 \%$ coniferous and $7.14 \%$ mixed. The habitats and communities have been evaluated for the species richness, native, endemic, economically important and threatened species. Based on these attributes habitats and communities have been prioritized. Among communities Abies pindrow - Pinus wallichiana mixed, Fraxinus xanthoxyloides, Picea smithiana - Pinus wallichiana mixed and Cedrus deodara-Acer cappadocicum mixed showed highest CPI and habitats forest, shady moist showed highest CPI value. Regular monitoring of the prioritized habitats and communities has been suggested. Also, mass scale propagation of native, endemic, economically important and threatened species and their plantation in the Lahaul valley have been suggested.
\end{abstract}

Key words: structure, composition, habitat, community, conservation, prioritization

\section{Introduction}

The Indian Himalayan Region (IHR) is very well known for its representative, natural, unique and socio-economically important plant diversity (Samant et al., 1998a). It is designated as one of the Biodiversity Hot Spots (Anonymous, 2007). It supports 18440 species of plants with 25-30\% of endemics (Samant et al., 1998a; Singh and Hajra, 1996). The inhabitants use this rich diversity for their sustenance. The increase in human population has increased the demand of economically important biodiversity elements. This has caused the over exploitation and habitat degradation of many economically important biodiversity elements and led the rapid loss of these elements. In view of the rapid loss of biodiversity elements, ecological and economical evaluation of the habitats and 
communities are essentially required. The review of literature indicates that in general, a large number of studies have been carried out on the flora, ecology, ethnobotany and rare endangered plants separately in the IHR (Aswal and Mehrotra, 1994; Chowdhery and Wadhwa, 1984; Dhaliwal and Sharma, 1999; Dhar et al., 1997; Kalakoti et al., 1986; Maity and Chauhan, 2002; Nautiyal et al., 1997; Rawal et al., 1994; Rawal and Pangtey, 1994; Rawat et al., 2001; Rawat et al., 1989; Rikhari et al., 1989; Samant and Joshi, 2004; Samant et al., 2002; Saxena and Singh, 1982; Singh and Singh, 1992; Singh and Rawat, 1999; Singh et al., 1996). In the IHR, a very few studies have been carried out to prioritize potential species and altitudinal zones (Dhar et al., 2000; Dhar and Samant, 1993; Pandey, 2006; Samant et al., 2007a; Samant and Pal, 2003; Samant et al., 2007b; Samant et al., 2007c), and habitats and communities (Arya, 2002; Joshi, 2002; Joshi and Samant, 2004; Pant and Samant, 2007; Samant et al., 2002). However, prioritization of habitats and communities based on species richness, native, endemic, economically important and threatened plants have been carried out by a few workers (Joshi and Samant, 2004; Pant and Samant, 2007). In Himachal Pradesh such integrated studies are not available. Therefore, present attempt has been made to; (i) assess the forest vegetation for community identification; (ii) evaluate the habitats and communities for species richness, native, endemic, economically important and threatened plants; (iii) prioritize habitats and communities for conservation; and (iv) suggest conservation measures.

\section{Materials and methods}

\section{Study area}

The study has been carried out during $2004-2007$ in Lahaul Valley $\left(32^{\circ} 22.517^{\prime} \mathrm{N}-32^{\circ}\right.$ 48.564'N Latitudes and $76^{\circ} 25.017^{\prime} \mathrm{E}-7^{\circ} 16.636^{\prime} \mathrm{E}$ Longitudes) of a proposed Cold Desert Biosphere Reserve (CDBR) (Fig. 1).

The climate varies from dry temperate to alpine types. The area remains snow covered almost for six months and receives average snowfall $120-400 \mathrm{~cm} \mathrm{year}^{-1}$, and average rainfall 10-300 mm year ${ }^{-1}$. The temperature ranges between $-19^{\circ}-32^{\circ} \mathrm{C}$ (Sinha and Samant, 2006). The highest mountain peak is Mulkila $(6,517 \mathrm{~m})$ and lowest point at Karunallah, (2 $400 \mathrm{~m}$ ) the entrance of Chenab into Pangi valley. The whole area of Lahaul is divided into three valleys namely, Chandra, Bhaga and Chandra-Bhaga. The rock system is very fragile and liable to erosion which is often accentuated by the rigorous of severe winters, avalanches and the strong winds that accompany them. The valley is inhabited by a large number of villages and the inhabitants are largely dependent on biodiversity elements for their sustenance.

\section{Survey, sampling, identification and data analysis}

Surveys were conducted to select sites and habitats along the Lahaul valley in forest zone between $2490-4000 \mathrm{~m}$ amsl. The habitats were identified on the basis of physical characters and dominance of vegetation. Attempts have been made to select sites and habitats on each and every accessible aspects. In each site, a plot of $50 \times 50 \mathrm{~m}$ was laid. 
Trees, saplings and seedlings were sampled by randomly placed 10, 10x10 m quadrats; shrubs by $20,5 \times 5 \mathrm{~m}$ quadrats; and herbs by $20,1 \times 1 \mathrm{~m}$ quadrats in each plot. For the collection of data from these quadrats standard ecological methods were followed (Curtis and Mc Intosh, 1950; Dhar et al., 1997; Greig-Smith, 1957; Misra, 1968; Mueller-Dombois and Ellenberge, 1974; Samant et al., 2002; Joshi and Samant, 2004). From each site, samples of each species were collected and identified with the help of floras (Aswal and Mehrotra, 1994; Chowdhery and Wadhwa, 1984; Dhaliwal and Sharma, 1999; Polunin and Stainton, 1984; Murti, 2001).

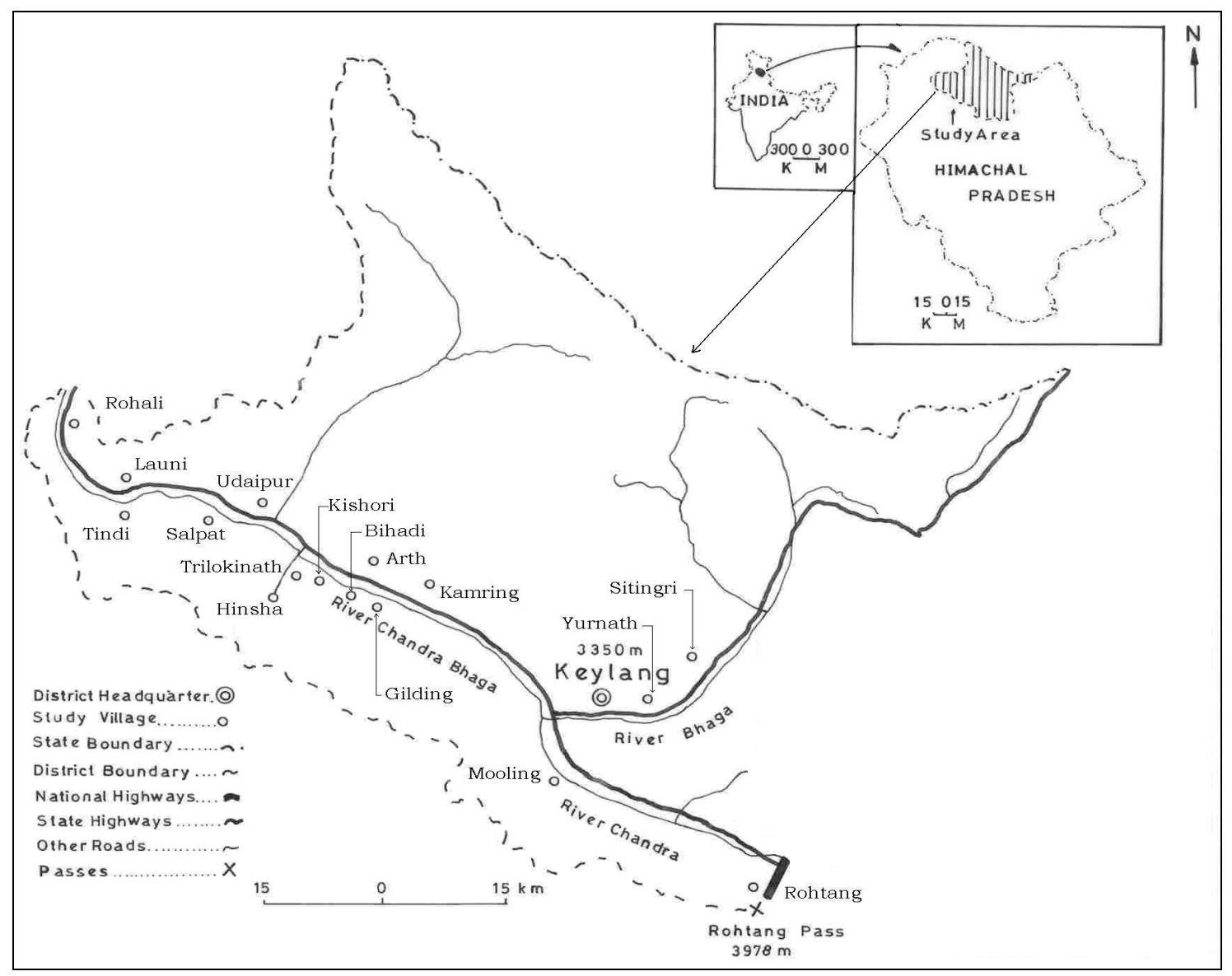

Figure 1. Study area

The forest communities were identified on the basis of IVI values of trees. The single tree species representing $>50 \%$ of the total IVI was designated as a single species dominated community, whereas two or more species contributing 50 or $>50 \%$ of the total IVI were named as a mixed community. Species richness was determined as the number of species. 


\section{Identification of native, endemic, economically important and threatened plants}

The species with its origin or first record from the Himalayan Region were considered as natives (Samant et al., 1998a; 2002). In case of Pteridophytes the endemic and nearendemic species have been considered as natives to the Himalayan region. The species restricted to IHR have been considered as endemic, whereas those extending their distribution to neighbouring Countries/States were considered as near-endemic (Dhar and Samant, 1993; Samant and Dhar, 1997; Samant et al., 1996a; 1998a).

The information on economically important species was generated through Participatory Rural Appraisal (PRA) (Samant et al., 2002; 2003) and the interviews of the knowledgeable persons including Amchies (local herbal doctors). Among the village experts, one person was hired to survey and collect the economically important species from wild habitats. Fresh samples of the useful species were collected and identified with the help of florulas (Aswal and Mehrotra, 1994; Chowdhery and Wadhwa, 1984; Polunin and Stainton, 1984).

The threatened species were identified based on habitat preference, distribution range, population size, use pattern, extraction trend, nativity and endemism of the species (Samant et al., 1996b; 1998b; 2002).

\section{Prioritization of habitats and communities}

The prioritization of habitats and communities has been done using eight parameters like species richness, economically important, native, endemic, threatened plants, altitude, site representation and habitats number following Joshi and Samant (2004); Pant and Samant (2007); Samant et al. (2002) (Table 1).

Table 1. Criteria's used for the prioritization of habitats and communities

\begin{tabular}{ccccccccc}
\hline Marks & $\begin{array}{c}\text { Richness } \\
(\boldsymbol{\%})\end{array}$ & $\begin{array}{c}\text { EIP } \\
(\boldsymbol{\%})\end{array}$ & $\begin{array}{c}\text { Native } \\
(\boldsymbol{\%})\end{array}$ & $\begin{array}{c}\text { Endemic } \\
(\boldsymbol{\%})\end{array}$ & $\begin{array}{c}\text { Threatened } \\
(\boldsymbol{\%})\end{array}$ & SR & $\begin{array}{c}\text { Altitude } \\
(\mathbf{m})\end{array}$ & $\begin{array}{c}\text { Habitats } \\
*\end{array}$ \\
\hline 10 & $>50$ & $>45$ & $>45$ & $>40$ & $>40$ & 1 & $<200$ & 1 \\
8 & $46-50$ & $41-45$ & $41-45$ & $36-40$ & $36-40$ & 2 & $200-400$ & 2 \\
6 & $41-45$ & $36-40$ & $36-40$ & $31-35$ & $31-35$ & 3 & $400-600$ & 3 \\
4 & $36-40$ & $31-35$ & $31-35$ & $26-30$ & $26-30$ & 4 & $600-800$ & 4 \\
2 & $<36$ & $<31$ & $<31$ & $<26$ & $<26$ & $>4$ & $>800$ & $>4$ \\
\hline
\end{tabular}

Abbreviations: EIP $=$ Economically Important Plants; SR $=$ Site representation; and $*=$ Criteria only applied for the communities

\section{Results}

\section{Habitat diversity}

Fifteen habitats (15) Rocky, Bouldary, Shady moist, Forest, Parasite, Degraded, Dry, Riverine, Water courses, Grassland, Marshy, Shrubberies, Near-settlements, Camping sites, and Road sides were identified (Table 2). The bouldary, dry, rocky, shady moist and grassland habitats showed wide range of distribution. The site representation varied from 316, Species richness ranged from 5-259, natives 3-104, endemics 0-87, economical important species 3-202, and threatened species 3-98. Amongst the habitats, maximum 
species richness (259), native (104), endemic (87), economically important (202), threatened (98) species were recorded in the forest habitat, followed by shady moist, species richness (235), native (94), endemic (84), economically important (191), threatened (93); dry habitat, species richness (170), native (69), endemic (42), economical important (134), threatened (45); grasslands, species richness (146), native (47), endemic (28), economical important (143), and threatened (29) species were recorded. The remaining habitats showed relatively less number of species (Table 2). The notable native, endemic, economically important and threatened species of the prioritized habitats have been presented in Table 3.

Table 2. Prioritization of habitats for conservation in Lahaul valley of the proposed CDBR

\begin{tabular}{lcccccccc}
\hline Habitat Type & SR & AD $(\mathbf{m})$ & SPR & N & En & EIP & TS & CPI \\
\hline Rocky & 15 & $2500-4000$ & 106 & 40 & 30 & 77 & 35 & 14 \\
Bouldary & 16 & $2490-4000$ & 88 & 24 & 18 & 62 & 28 & 14 \\
Shady Moist & 15 & $2400-4000$ & 235 & 94 & 84 & 191 & 93 & 38 \\
Forest & 14 & $2500-4000$ & 259 & 104 & 87 & 202 & 98 & 44 \\
Parasitic & 3 & $2500-3690$ & 5 & 3 & 3 & 3 & 4 & 14 \\
Degraded & 12 & $2500-4000$ & 56 & 12 & 11 & 44 & 10 & 14 \\
Dry & 15 & $2490-4000$ & 170 & 69 & 42 & 134 & 45 & 14 \\
Riverine & 14 & $2500-4000$ & 71 & 23 & 19 & 60 & 17 & 14 \\
Water Courses & 11 & $2490-4000$ & 21 & 9 & 5 & 14 & 6 & 14 \\
Grassland & 15 & $2400-4000$ & 146 & 47 & 28 & 143 & 29 & 14 \\
Marshy & 11 & $2500-3855$ & 17 & 4 & - & 11 & 3 & 14 \\
Shrubberries & 14 & $2500-4000$ & 45 & 15 & 11 & 38 & 14 & 14 \\
Near Settlements & 13 & $2490-4000$ & 52 & 12 & 10 & 50 & 11 & 14 \\
Camping Sites & 14 & $2500-4000$ & 51 & 13 & 8 & 49 & 3 & 14 \\
Road Sides & 12 & $2500-3900$ & 60 & 14 & 11 & 58 & 13 & 14 \\
\hline
\end{tabular}

Abbreviations: SR = Site representation; AD = Altitudinal Distribution; EIP = Economically Important Plants; $\mathrm{CPI}=$ Conservation Priority Index; SPR = Species Richness; $\mathrm{N}=$ Native; En = Endemic; and TS $=$ Threatened Species

\section{Community diversity, species composition and structural pattern}

Overall 96 sites representing 8 aspects and 15 habitats were sampled and 14 tree communities from forests zone were identified (Table 4). The identified communities were broad leaved deciduous (i.e., Betula utilis, Hippophae salicifolia, Juglans regia - Ulmus wallichian - Acer acuminatum mixed, Salix daphnoides and Fraxinus xanthoxyloides); evergreen coniferous and deciduous broad leaved mixed (i.e., Cedrus deodara - Acer cappadocicum mixed), and coniferous evergreen (i.e., Abies pindrow, Abies pindrow Pinus wallichiana mixed, Cedrus deodara, Juniperus polycarpos, Juniperus polycarpos Cedrus deodara mixed, Picea smithiana, Picea smithiana - Pinus wallichiana mixed and Pinus wallichiana) communities. The communities having relatively wide altitudinal range of distribution were Juniperus polycarpos, Pinus wallichiana, Cedrus deodara and Picea smithiana (Table 4). 
Table 3. Some important native, endemic, near-endemic, economically important and threatened species of the main prioritized habitat

\begin{tabular}{|c|c|c|c|c|}
\hline $\begin{array}{c}\text { Prioritized } \\
\text { habitats }\end{array}$ & Native & $\begin{array}{c}\text { Endemic/Near- } \\
\text { Endemic }\end{array}$ & $\begin{array}{c}\text { Economically } \\
\text { important }\end{array}$ & Threatened \\
\hline Forests & $\begin{array}{l}\text { Selinum coniifolium, } \\
\text { Campanula aristata, } \\
\text { Cyananthus lobatus, } \\
\text { Rhododendron } \\
\text { campanulatum, } \\
\text { Picrorhiza kurrooa }\end{array}$ & $\begin{array}{l}\text { Allium stracheyi, } \\
\text { Berberis } \\
\text { pseudumbellata, } \\
\text { Campanula } \\
\text { cashmeriana, } \\
\text { Dactylorhiza } \\
\text { hatagirea }\end{array}$ & $\begin{array}{l}\text { Carum carvi, } \\
\text { Heracleum candicans, } \\
\text { Hippophae rhamnoides, } \\
\text { Corydalis govaniana, } \\
\text { Gentiana coronata, } \\
\text { Juglans regia, Rheum } \\
\text { webbianum, Abies } \\
\text { pindrow }\end{array}$ & $\begin{array}{l}\text { Allium stracheyi, } \\
\text { Heracleum thomsonii, } \\
\text { Selinum coniifolium, } \\
\text { Lonicera spinosa, } \\
\text { Dioscorea deltoidea, } \\
\text { Gentianella } \\
\text { moorcroftiana, } \\
\text { Swertia alternifolia, } \\
\text { Polygonatum } \\
\text { cirrhifolium, } \\
\text { Aconitum } \\
\text { heterophyllum, } \\
\text { Juniperus indica }\end{array}$ \\
\hline $\begin{array}{l}\text { Shady } \\
\text { Moist }\end{array}$ & $\begin{array}{l}\text { Acer acuminatum, } \\
\text { Angelica glauca, } \\
\text { Bupleurum candollii, } \\
\text { Chaerophyllum } \\
\text { villosum, Indigofera } \\
\text { heterantha }\end{array}$ & $\begin{array}{l}\text { Acer acuminatum, } \\
\text { Chaerophyllum } \\
\text { villosum, Erigeron } \\
\text { bellidioides, } \\
\text { Codonopsis } \\
\text { clematidea }\end{array}$ & $\begin{array}{l}\text { Allium carolinianum, } \\
\text { Angelica glauca, } \\
\text { Chaerophyllum } \\
\text { reflexum, Corylus } \\
\text { jacquemontii, Cedrus } \\
\text { deodara, Taxus baccata } \\
\text { subsp. wallichiana }\end{array}$ & $\begin{array}{l}\text { Acer acuminatum, } \\
\text { Allium victorialis, } \\
\text { Bunium persicum, } \\
\text { Inula grandiflora, } \\
\text { Saussurea } \\
\text { glanduligera, Onosma } \\
\text { hispida, Lilium } \\
\text { polyphyllum }\end{array}$ \\
\hline Dry & $\begin{array}{l}\text { Bupleurum } \\
\text { lanceolatum, Cortia } \\
\text { depressa, Ligusticum } \\
\text { elatum, Anaphalis } \\
\text { busua, Eremurus } \\
\text { himalaicus }\end{array}$ & $\begin{array}{l}\text { Bupleurum } \\
\text { lanceolatum, } \\
\text { Heracleum } \\
\text { thomsonii, Berberis } \\
\text { jaeschkeana, Cortia } \\
\text { depressa, Aster } \\
\text { indamellus, } \\
\text { Echinops cornigerus }\end{array}$ & $\begin{array}{l}\text { Bunium persicum, } \\
\text { Bupleurum falcatum, } \\
\text { Ferula jaeschkeana, } \\
\text { Caragana versicolor, } \\
\text { Ribes alpestre, Ajuga } \\
\text { bracteosa }\end{array}$ & $\begin{array}{l}\text { Saussurea deltoidea, } \\
\text { Vincetoxicum } \\
\text { hirundinaria, Lonicera } \\
\text { hypoleuca, Datisca } \\
\text { cannabina, Syringa } \\
\text { emodi, Hyoscyamus } \\
\text { niger, Juniperus } \\
\text { polycarpos }\end{array}$ \\
\hline Bouldary & $\begin{array}{l}\text { Cirsium verutum, } \\
\text { Gnaphalium thomsonii, } \\
\text { Hackelia uncinata, } \\
\text { Rhodiola tibetica, } \\
\text { Hedysarum } \\
\text { astragaloides, } \\
\text { Poa koelzii }\end{array}$ & $\begin{array}{l}\text { Cirsium verutum, } \\
\text { Silene } \\
\text { moorcroftiana, } \\
\text { Rhodiola } \\
\text { heterodonta, } \\
\text { Oryzopsis lateralis, } \\
\text { Physochlaena } \\
\text { praealta, Cystopteris } \\
\text { montana }\end{array}$ & $\begin{array}{l}\text { Cardamine impatiens, } \\
\text { Nepeta eriostachya, } \\
\text { Podophyllum } \\
\text { hexandrum, Rheum } \\
\text { australe, Bergenia } \\
\text { stracheyi, Ephedra } \\
\text { gerardiana, Adiantum } \\
\text { capillus-veneris }\end{array}$ & $\begin{array}{l}\text { Silene moorcroftiana, } \\
\text { Hypericum } \\
\text { perforatum, } \\
\text { Meconopsis aculeata, } \\
\text { Podophyllum } \\
\text { hexandrum, Bergenia } \\
\text { ligulata, Physochlaena } \\
\text { praealta, Viola } \\
\text { biflora, Athyrium } \\
\text { davidii }\end{array}$ \\
\hline Degraded & $\begin{array}{l}\text { Cousinia thomsonii, } \\
\text { Astragalus rhizanthus, } \\
\text { Rubus cordifolius, } \\
\text { Bupleurum } \\
\text { lanceolatum, } \\
\text { Ligusticum elatum, } \\
\text { Senecio } \\
\text { krascheninnikovii }\end{array}$ & $\begin{array}{l}\text { Astragalus bicuspis, } \\
\text { Agrostis pilosula, } \\
\text { Bupleurum } \\
\text { lanceolatum, } \\
\text { Cirsium wallichii, } \\
\text { Echinops cornigerus }\end{array}$ & $\begin{array}{l}\text { Cousinia thomsonii, } \\
\text { Medicago falcata, } \\
\text { Melilotus officinalis, } \\
\text { Thymus linearis, } \\
\text { Plantago depressa, } \\
\text { Prunus armeniaca, } \\
\text { Verbascum thapsus, } \\
\text { Pinus wallichiana }\end{array}$ & $\begin{array}{l}\text { Hyssopus officinalis, } \\
\text { Bupleurum } \\
\text { lanceolatum, Arctium } \\
\text { lappa, Artemisia } \\
\text { maritima, Juniperus } \\
\text { polycarpos, Solanum } \\
\text { nigrum }\end{array}$ \\
\hline
\end{tabular}


Amongst the communities, tree density was maximum for Hippophae salicifolia community (1850.00 Ind ha $\left.{ }^{-1}\right)$, followed by Fraxinus xanthoxyloides (1000.00 Ind ha $\left.{ }^{-1}\right)$, Juglans regia - Ulmus wallichiana - Acer acuminatum mixed (760.00 Ind ha ${ }^{-1}$ ), Abies pindrow - Pinus wallichiana mixed (640.00 Ind ha ${ }^{-1}$ ), Juniperus polycarpos - Cedrus deodara mixed (600.00 Ind ha $\left.{ }^{-1}\right)$. Cedrus deodara - Acer cappadocicum mixed $\left(170.54 \mathrm{~m}^{2}\right.$ $\left.\mathrm{ha}^{-1}\right)$ community had lowest density. The total basal area $\left(124.89 \mathrm{~m}^{2} \mathrm{ha}^{-1}\right)$ was maximum for Abies pindrow - Pinus wallichiana mixed community, followed by Juniperus polycarpos - Cedrus deodara mixed (110.64 $\left.\mathrm{m}^{2} \mathrm{ha}^{-1}\right)$, Picea smithiana $\left(92.26 \mathrm{~m}^{2} \mathrm{ha}^{-1}\right)$, and Juglans regia - Ulmus wallichiana - Acer acuminatum mixed $\left(91.23 \mathrm{~m}^{2} \mathrm{ha}^{-1}\right)$. Juniperus polycarpos $\left(13.65 \mathrm{~m}^{2} \mathrm{ha}^{-1}\right)$ community showed lowest total basal area. Total shrub density was highest in Cedrus deodara (2611.7 Ind ha ${ }^{-1}$ ) community, followed by Hippophae salicifolia (2520.00 Ind ha ${ }^{-1}$ ), Betula utilis (2230.00 Ind ha ${ }^{-1}$ ) and Abies pindrow (1994.00 Ind $\left.\mathrm{ha}^{-1}\right)$. Pinus wallichiana community showed the highest herb density $\left(95.10 \mathrm{Ind} \mathrm{m}^{-2}\right)$, followed by Hippophae salicifolia (63.85 $\mathrm{Ind} \mathrm{m}^{-2}$ ), Juniperus polycarpos $\left(32.77 \mathrm{Ind} \mathrm{m}^{-2}\right.$ ) and Picea smithiana (32.40 Ind $\mathrm{m}^{-2}$ ) communities. Fraxinus xanthoxyloides community showed the maximum seedlings density (1300.00 Ind $\mathrm{ha}^{-1}$ ), followed by Abies pindrow (557.18 Ind $\mathrm{ha}^{-1}$ ) and Abies pindrow - Pinus wallichiana mixed (390.00 Ind ha ${ }^{-1}$ ) communities. Highest saplings density was recorded for Cedrus deodara community (816.42 Ind $\mathrm{ha}^{-1}$ ), followed by Hippophae salicifolia (790.00 Ind ha ${ }^{-1}$ ), Juniperus polycarpos (750.58 Ind ha $\left.{ }^{-1}\right)$, Juglans regia - Ulmus wallichiana-Acer acuminatum mixed (660.00 Ind ha ${ }^{-1}$ ) communities (Table 4).

Table 4. Community types, distribution pattern, structural pattern and major tree associates in Lahaul valley of the Proposed CDBR

\begin{tabular}{|c|c|c|c|c|}
\hline Community types & SR & Altitudinal range (m) & Habitat type (s) & Slope $\left({ }^{\circ}\right)$ \\
\hline Juniperus polycarpos & 43 & $2760-3782$ & $\mathrm{~A}, \mathrm{~B}, \mathrm{C}, \mathrm{D}, \mathrm{E}, \mathrm{F}$ & $35-60$ \\
\hline Pinus wallichiana & 13 & $2845-3518$ & B, C, D, E, F & $35-50$ \\
\hline Cedrus deodara & 11 & $2550-2830$ & $\mathrm{~A}, \mathrm{E}, \mathrm{C}, \mathrm{D}, \mathrm{F}$ & $20-60$ \\
\hline Abies pindrow & 6 & $3220-3440$ & $\mathrm{~A}, \mathrm{D}, \mathrm{F}$ & $40-55$ \\
\hline Picea smithiana & 8 & $2742-3100$ & $\mathrm{~A}, \mathrm{~B}, \mathrm{D}, \mathrm{F}$ & $40-50$ \\
\hline Betula utilis & 5 & $3440-3855$ & $A, D, E, F$ & $55-70$ \\
\hline $\begin{array}{l}\text { Juniperus polycarpos - } \\
\text { Cedrus deodara mixed }\end{array}$ & 2 & $2760-2780$ & $A, B$ & $35-50$ \\
\hline Hippophae salicifolia & 1 & 3000 & $\mathrm{~L}$ & 35 \\
\hline $\begin{array}{l}\text { Abies pindrow -Pinus } \\
\text { wallichiana mixed }\end{array}$ & 1 & 3268 & $\mathrm{D}$ & 65 \\
\hline $\begin{array}{l}\text { Juglans regia - Ulmus } \\
\text { wallichiana - Acer } \\
\text { acuminatum mixed }\end{array}$ & 1 & 2490 & D & 25 \\
\hline $\begin{array}{l}\text { Picea smithiana - Pinus } \\
\text { wallichiana mixed }\end{array}$ & 2 & $2510-2650$ & D & $50-60$ \\
\hline Salix daphnoides & 1 & 2560 & A & 55 \\
\hline $\begin{array}{l}\text { Cedrus deodara - Acer } \\
\text { cappadocicum mixed }\end{array}$ & 1 & 2560 & A & 60 \\
\hline $\begin{array}{l}\text { Fraxinus } \\
\text { xanthoxyloides }\end{array}$ & 1 & 2580 & A & 65 \\
\hline
\end{tabular}


Table 4. cont.

\begin{tabular}{|c|c|c|c|c|c|c|c|}
\hline \multirow[t]{2}{*}{ Community types } & \multicolumn{5}{|c|}{ Density $\left(\right.$ Ind ha $\left.{ }^{-1}\right)$} & \multirow{2}{*}{$\begin{array}{l}\text { TBA } \\
\left(m^{2} h^{-1}\right)\end{array}$} & \multirow{2}{*}{$\begin{array}{l}\text { Major Associate } \\
\text { Species }\end{array}$} \\
\hline & Trees & Seedlings & Saplings & Shrubs & Herbs & & \\
\hline Juniperus polycarpos & 448.55 & 89.03 & 750.58 & 696.24 & 32.77 & 13.65 & $\begin{array}{l}\text { Picea smithiana \& } \\
\text { Pinus wallichiana }\end{array}$ \\
\hline Pinus wallichiana & 456.01 & 222.05 & 362.42 & 1178.97 & 95.10 & 32.46 & $\begin{array}{l}\text { Juniperus polycarpos } \\
\& \text { Betula utilis }\end{array}$ \\
\hline Cedrus deodara & 422.12 & 178.75 & 816.42 & 2611.70 & 28.24 & 65.14 & $\begin{array}{l}\text { Juniperus } \\
\text { polycarpos, Robinia } \\
\text { pseudoacacia, Pinus } \\
\text { wallichiana, Picea } \\
\text { smithiana, Acer } \\
\text { acuminat. \& Celtis } \\
\text { australis }\end{array}$ \\
\hline Abies pindrow & 475.79 & 557.18 & 162.15 & 1994.00 & 22.03 & 61.43 & $\begin{array}{l}\text { Pinus wallichiana \& } \\
\text { Betula utilis }\end{array}$ \\
\hline Picea smithiana & 399.76 & 290.52 & 337.26 & 767.20 & 32.40 & 92.26 & $\begin{array}{l}\text { Pinus wallichiana, } \\
\text { Juniperus } \\
\text { polycarpos, Taxus } \\
\text { baccata subsp. } \\
\text { wallichiana \& } \\
\text { Corylus jacquemontii }\end{array}$ \\
\hline Betula utilis & 588.52 & 136.30 & 330.00 & 2230.00 & 30.48 & 55.15 & $\begin{array}{l}\text { Pinus wallichiana \& } \\
\text { Abies pindrow }\end{array}$ \\
\hline $\begin{array}{l}\text { Juniperus polycarpos } \\
\text { - Cedrus deodara } \\
\text { mixed }\end{array}$ & 600.00 & 216.67 & 566.67 & 1290.00 & 26.65 & 110.64 & $\begin{array}{l}\text { Pinus wallichiana, } \\
\text { Juglans regia \& } \\
\text { Pyrus pashia }\end{array}$ \\
\hline $\begin{array}{l}\text { Hippophae } \\
\text { salicifolia }\end{array}$ & 1850.00 & - & 790.00 & 2520.00 & 63.85 & 22.07 & Rosa webbiana \\
\hline $\begin{array}{l}\text { Abies pindrow - } \\
\text { Pinus wallichiana } \\
\text { mixed }\end{array}$ & 640.00 & 390.00 & 310.00 & 545.00 & 30.96 & 124.89 & Picea smithiana \\
\hline $\begin{array}{l}\text { Juglans regia -Ulmus } \\
\text { wallichiana - Acer } \\
\text { acuminatum mixed }\end{array}$ & 760.00 & - & 660.00 & 490.00 & 23.77 & 91.23 & $\begin{array}{l}\text { Ulmus villosa, } \\
\text { Prunus cornuta, } \\
\text { Fraxinus micrantha, } \\
\text { Acer cappadoc, } \\
\text { Corylus jacquemontii } \\
\& \text { Pinus wallichiana }\end{array}$ \\
\hline $\begin{array}{l}\text { Picea smithiana - } \\
\text { Pinus wallichiana } \\
\text { mixed }\end{array}$ & 305.56 & 290.00 & 310.00 & 1900.00 & 20.11 & 69.18 & $\begin{array}{l}\text { Abies pindrow, Picea } \\
\text { smithiana, Pinus } \\
\text { wallich. Acer } \\
\text { acuminatum \& } \\
\text { Corylus jacquemontii }\end{array}$ \\
\hline Salix daphnoides & 220.00 & - & 500.00 & & 23.67 & 25.33 & Cedrus deodara \\
\hline $\begin{array}{l}\text { Cedrus deodara - } \\
\text { Acer cappadocicum } \\
\text { mixed }\end{array}$ & 170.00 & - & - & 690.00 & 17.18 & 36.23 & Celtis australis \\
\hline $\begin{array}{l}\text { Fraxinus } \\
\text { xanthoxyloid. }\end{array}$ & 1000.00 & 1300.00 & 300.00 & 480.00 & 7.83 & 46.97 & Corylus jacquemontii \\
\hline
\end{tabular}

Abbreviations: $\mathrm{m}=$ Meters; Ind = Individual; $\mathrm{ha}^{-1}=$ Per hectare; $\mathrm{A}=$ Bouldary; $\mathrm{B}=$ Degraded $\mathrm{C}=\mathrm{Dry}$ slopes; $\mathrm{D}=$ Shady moist slopes; $\mathrm{E}=$ Riverine $; \mathrm{F}=$ Rocky; $\mathrm{L}=$ Grassland and $\mathrm{SR}=$ Site representation 


\section{Species richness}

The species richness within the identified communities for trees ranged from 1-9, shrubs 3-23, herbs 9-213, seedlings 0-5, and saplings 0-4. The richness of trees was highest in Juglans regia - Ulmus wallichiana - Acer acuminatum mixed community (i.e., 9), followed by Picea smithiana - Pinus wallichiana mixed community (8). The richness of shrubs was highest in Juniperus polycarpos community (23), followed by Cedrus deodara (18) and Pinus wallichiana (15) communities, whereas richness of herbs was again highest in Juniperus polycarpos community (213), followed by Pinus wallichiana (153) and Cedrus deodara (92) communities (Table 4).

\section{Native, endemic, economically important and threatened species}

The native species ranged from 7-90, endemic species 6-63, economically important species 15-194 and threatened species 6-47 within the communities. Maximum species were recorded in Juniperus polycarpos community (native 90; endemic 63; economically important 194), followed by Pinus wallichiana (native 81; endemic 61; economically important 147), Cedrus deodara (native 52; endemic 30; economically important 101), Picea smithiana (native 40; endemic 16; economically important 69) and Betula utilis (native 30; endemic 26; economically important 52) communities. The remaining communities showed comparatively less native and endemic species (Table 5).

Table 5. Prioritization of forest communities for conservation using different parameters in the Lahaul valley of Proposed CDBR

\begin{tabular}{|c|c|c|c|c|c|c|c|c|}
\hline Community Type & Habitat(s) & SR & $\mathbf{A D}(\mathbf{m})$ & SPR & $\mathbf{N}$ & En & EIP & TS \\
\hline Juniperus polycarpos & 6 & 43 & $2760-3700$ & 242 & 90 & 63 & 194 & 47 \\
\hline Pinus wallichiana & 5 & 13 & $2845-3500$ & 173 & 81 & 61 & 147 & 46 \\
\hline Cedrus deodara & 5 & 11 & $2550-2830$ & 117 & 52 & 30 & 101 & 33 \\
\hline Abies pindrow & 3 & 6 & $3220-3440$ & 57 & 30 & 16 & 49 & 15 \\
\hline Picea smithiana & 4 & 8 & $2742-3100$ & 83 & 40 & 16 & 69 & 20 \\
\hline Betula utilis & 4 & 5 & $3440-3855$ & 59 & 30 & 26 & 52 & 17 \\
\hline $\begin{array}{l}\text { Juniperus polycarpos-Cedrus } \\
\text { deodara mixed }\end{array}$ & 2 & 2 & $2760-2780$ & 39 & 12 & 11 & 36 & 12 \\
\hline Hippophae salicifolia & 1 & 1 & $3000-3100$ & 30 & 13 & 9 & 24 & 7 \\
\hline $\begin{array}{l}\text { Abies pindrow-Pinus } \\
\text { wallichiana } \text { mixed }\end{array}$ & 1 & 1 & $3260-3340$ & 24 & 14 & 10 & 21 & 9 \\
\hline $\begin{array}{l}\text { Picea smithiana-Pinus } \\
\text { wallichiana mixed }\end{array}$ & 1 & 2 & $2490-2600$ & 46 & 16 & 18 & 44 & 22 \\
\hline $\begin{array}{l}\text { Juglans regia-Ulmus } \\
\text { wallichiana-Acer acuminatum } \\
\text { mixed }\end{array}$ & 1 & 1 & $2450-2550$ & 50 & 15 & 13 & 42 & 23 \\
\hline $\begin{array}{l}\text { Cedrus deodara-Acer } \\
\text { cappadocicum mixed }\end{array}$ & 1 & 1 & $2560-2630$ & 26 & 9 & 8 & 23 & 13 \\
\hline Salix daphnoides & 1 & 1 & $2560-2620$ & 28 & 8 & 6 & 24 & 7 \\
\hline Fraxinus xanthoxyloides & 1 & 1 & $2500-2650$ & 15 & 7 & 6 & 15 & 6 \\
\hline
\end{tabular}


Amongst the communities, threatened species ranged from 6-47 (Table 5). It was highest in Juniperus polycarpos community (Endangered 1; Vulnerable 11; Near Threatened 35), followed by Pinus wallichiana (Critically Endangered 2; Endangered 5; Vulnerable 13; Near Threatened 26), Cedrus deodara (Critically Endangered 2; Endangered 2; Vulnerable 7; Near Threatened 22), Juglans regia-Ulmus wallichiana-Acer acuminatum mixed (Critically Endangered 1; Endangered 8; Vulnerable 6; Near Threatened 8), Picea smithiana-Pinus wallichiana mixed (Critically Endangered 1; Endangered 2; Vulnerable 7; Near Threatened 12) and Picea smithiana (Vulnerable 2; Near Threatened 18) communities. The remaining communities showed comparatively less number for threatened species. The notable native, endemic, economically important and threatened species of the prioritized communities have been presented in Table 6.

\section{Prioritization of habitats and forest communities}

Amongst habitats, the forest habitat showed highest (i.e., 44) Conservation Priority Index (CPI), followed by shady moist (CPI: 38) habitat. The remaining habitats showed CPI 14, each (Table 2).

Among the communities, Abies pindrow - Pinus wallichiana mixed community showed highest i.e., 70 Conservation Priority Index (CPI), followed by Fraxinus xanthoxyloides (68) and Picea smithiana - Pinus wallichiana mixed and Cedrus deodara - Acer cappadocicum mixed (62, each) communities. However, lowest CPI (38, each) was recorded for Juniperus polycarpos and Cedrus deodara communities (Table 1; Figure 2).

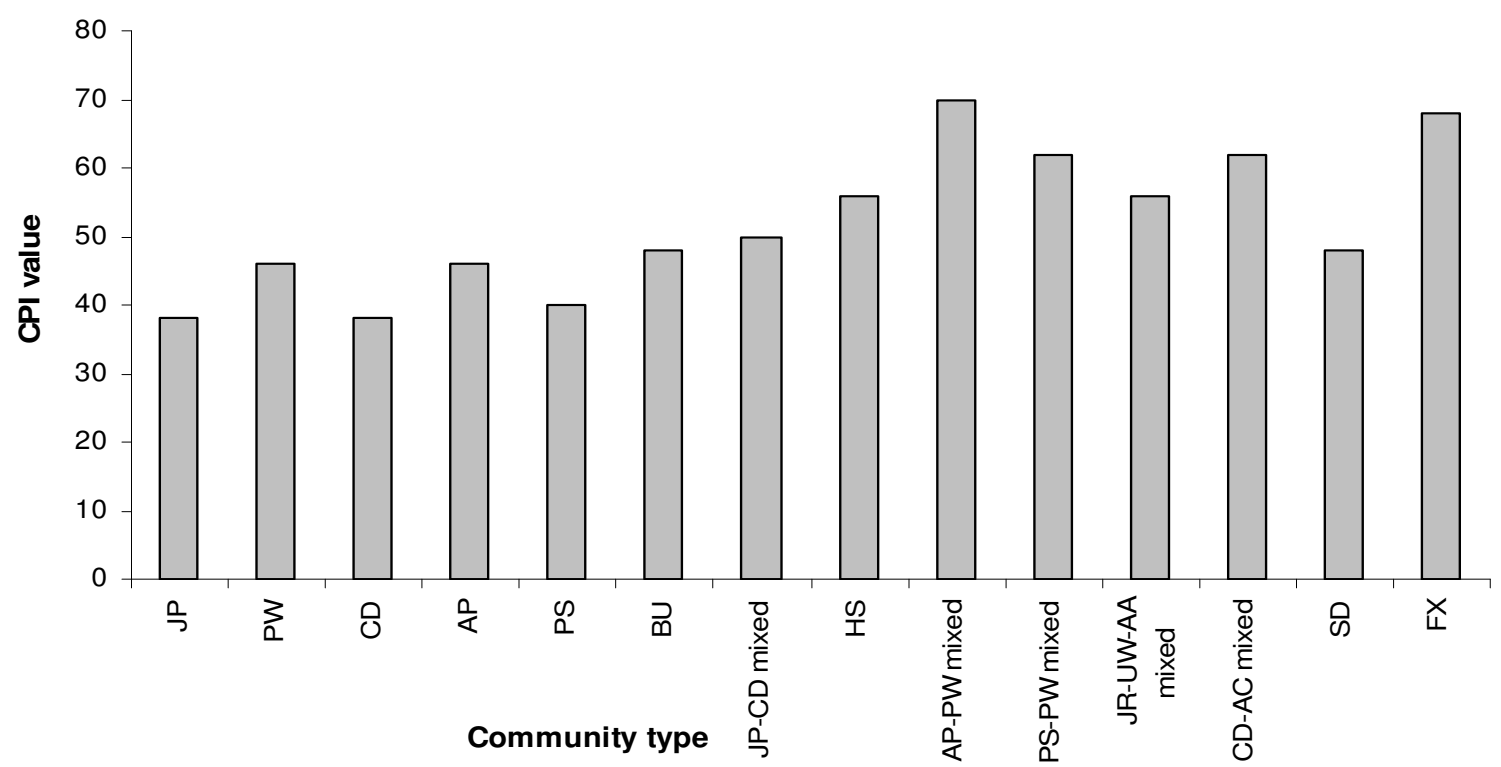

Figure 2. Conservation Prioritization Index of forest communities in Lahaul valley of the Proposed CDBR 
Table 6. Some important native, endemic, near-endemic, economically important and threatened species of the prioritized communities

\begin{tabular}{|c|c|c|c|c|}
\hline $\begin{array}{c}\text { Prioritized } \\
\text { communities }\end{array}$ & Native & $\begin{array}{c}\begin{array}{c}\text { Endemic/near- } \\
\text { endemic }\end{array} \\
\end{array}$ & Economically important & Threatened \\
\hline $\begin{array}{l}\text { Abies pindrow- } \\
\text { Pinus } \\
\text { wallichiana } \\
\text { mixed }\end{array}$ & $\begin{array}{l}\text { Cousinia thomsonii, } \\
\text { Pedicularis porrecta, } \\
\text { Plantago himalaica, } \\
\text { Lonicera obovata, } \\
\text { Rosa webbiana, } \\
\text { Rubus foliolosus, } \\
\text { Viburnum } \\
\text { cotinifolium }\end{array}$ & $\begin{array}{l}\text { Bergenia } \\
\text { stracheyi, Galium } \\
\text { asperuloides, } \\
\text { Lonicera obovata, } \\
\text { Pinus wallichiana, } \\
\text { Abies pindrow }\end{array}$ & $\begin{array}{l}\text { Viola biflora, Polygonatum } \\
\text { multiflorum, } \\
\text { Fragaria vesca var. } \\
\text { nubicola, } \\
\text { Eragrostis minor, Dactylis } \\
\text { glomerata, Cousinia } \\
\text { thomsonii }\end{array}$ & $\begin{array}{l}\text { Polygonatum } \\
\text { multiflorum, } \\
\text { Plantago himalaica, } \\
\text { Pedicularis porrecta, } \\
\text { Bergenia stracheyi }\end{array}$ \\
\hline $\begin{array}{l}\text { Fraxinus } \\
\text { xanthoxyloides }\end{array}$ & $\begin{array}{l}\text { Cremanthodium } \\
\text { arnicoides, } \\
\text { Stachys } \\
\text { melissaefolia, } \\
\text { Indigofera } \\
\text { hebepetala, } \\
\text { Lonicera spinosa, } \\
\text { Fraxinus } \\
\text { xanthoxyloides }\end{array}$ & $\begin{array}{l}\text { Stachys } \\
\text { melissaefolia, } \\
\text { Indigofera } \\
\text { hebepetala, } \\
\text { Lonicera spinosa, } \\
\text { Fraxinus } \\
\text { xanthoxyloides }\end{array}$ & $\begin{array}{l}\text { Artemisia gmelinii, Carum } \\
\text { carvi, Chenopodium } \\
\text { hybridum, Galinsoga } \\
\text { parviflora, Origanum } \\
\text { vulgare, Verbascum } \\
\text { thapsus, Sorbaria } \\
\text { tomentosa, Rosa webbiana }\end{array}$ & $\begin{array}{l}\text { Carum carvi, } \\
\text { Indigofera } \\
\text { hebepetala, Lonicera } \\
\text { spinosa, Fraxinus } \\
\text { micrantha }\end{array}$ \\
\hline $\begin{array}{l}\text { Picea smithiana- } \\
\text { Pinus } \\
\text { wallichiana } \\
\text { mixed }\end{array}$ & $\begin{array}{l}\text { Chaerophyllum } \\
\text { reflexum, } \\
\text { Cremanthodium } \\
\text { arnicoides, } \\
\text { Impatiens } \\
\text { glandulifera, } \\
\text { Cotoneaster obtusus, } \\
\text { Lonicera hypoleuca, } \\
\text { Salix denticulata }\end{array}$ & $\begin{array}{l}\text { Bergenia } \\
\text { stracheyi, } \\
\text { Bilderdykia } \\
\text { pterocarpa, } \\
\text { Impatiens } \\
\text { glandulifera, } \\
\text { Rubia cordifolia, } \\
\text { Indigofera } \\
\text { hebepetala, } \\
\text { Syringa emodi, } \\
\text { Viburnum } \\
\text { cotinifolium, } \\
\text { Picea smithiana }\end{array}$ & $\begin{array}{l}\text { Adiantum venustum, } \\
\text { Artemisia dracunculus, } \\
\text { Asparagus filicinus, } \\
\text { Fragaria nubicola, } \\
\text { Podophyllum hexandrum, } \\
\text { Viola pilosa, } \\
\text { Jasminum humile, Ribes } \\
\text { alpestre, } \\
\text { Corylus jacquemontii }\end{array}$ & $\begin{array}{l}\text { Asparagus filicinus, } \\
\text { Bilderdykia } \\
\text { pterocarpa, } \\
\text { Dioscorea deltoidea, } \\
\text { Podophyllum } \\
\text { hexandrum, } \\
\text { Polygonatum } \\
\text { multiflorum, } \\
\text { Juniperus } \\
\text { polycarpos, Taxus } \\
\text { baccata subsp. } \\
\text { wallichiana }\end{array}$ \\
\hline $\begin{array}{l}\text { Cedrus deodara- } \\
\text { Acer } \\
\text { cappadocicum } \\
\text { mixed }\end{array}$ & $\begin{array}{l}\text { Anaphalis busua, } \\
\text { Thalictrum } \\
\text { cultratum, } \\
\text { Plectranthus } \\
\text { rugosus, Rosa } \\
\text { webbiana, Spiraea } \\
\text { canescens }\end{array}$ & $\begin{array}{l}\text { Stachys } \\
\text { melissaefolia, } \\
\text { Lonicera spinosa, } \\
\text { Spiraea } \\
\text { canescens, } \\
\text { Cedrus deodara, } \\
\text { Celtis australis }\end{array}$ & $\begin{array}{l}\text { Artemisia maritima, Carum } \\
\text { carvi, } \\
\text { Dioscorea deltoidea, } \\
\text { Elsholtzia ciliata, } \\
\text { Geranium pratense, } \\
\text { Sonchus oleraceus, } \\
\text { Thymus linearis, } \\
\text { Plectranthus rugosus, } \\
\text { Sorbaria tomentosa }\end{array}$ & $\begin{array}{l}\text { Viola sylvatica, } \\
\text { Lonicera spinosa, } \\
\text { Rubus ellipticus, } \\
\text { Spiraea canescens, } \\
\text { Acer cappadocicum, } \\
\text { Celtis australis }\end{array}$ \\
\hline $\begin{array}{l}\text { Hippophae } \\
\text { salicifolia }\end{array}$ & $\begin{array}{l}\text { Aster molliusculus, } \\
\text { Astragalus } \\
\text { rhizanthus, } \\
\text { Heracleum } \\
\text { thomsonii, } \\
\text { Impatiens tingens, } \\
\text { Nepeta nervosa, } \\
\text { Nepeta laevigata, } \\
\text { Silene edgeworthii, } \\
\text { Synotis kunthiana }\end{array}$ & $\begin{array}{l}\text { Astragalus } \\
\text { rhizanthus, } \\
\text { Heracleum } \\
\text { thomsonii, } \\
\text { Phlomis } \\
\text { bracteosa, } \\
\text { Physochlaena } \\
\text { praealta, Silene } \\
\text { indica, Thalictrum } \\
\text { minus, Hippophae } \\
\text { salicifolia }\end{array}$ & $\begin{array}{l}\text { Artemisia parviflora, } \\
\text { Achillea millefolium, } \\
\text { Carum carvi, } \\
\text { Chaerophyllum reflexum, } \\
\text { Equisetum arvens, Phlomis } \\
\text { bracteosa, Rumex acetosa, } \\
\text { Silene indica, } \\
\text { Sonchus wightianus, } \\
\text { Scorzonera virgata, } \\
\text { Taraxacum officinale, } \\
\text { Urtica dioica, Hippophae } \\
\text { rhamnoides }\end{array}$ & $\begin{array}{l}\text { Carum carvi, } \\
\text { Heracleum } \\
\text { thomsonii, } \\
\text { Heracleum } \\
\text { candicans, Nepeta } \\
\text { nervosa, } \\
\text { Physochlaena } \\
\text { praealta, Thalictrum } \\
\text { minus, Hippophae } \\
\text { rhamnoides }\end{array}$ \\
\hline
\end{tabular}




\section{Discussion}

Conservation prioritization of the habitats, species and communities is pre-requisite for the management planning of the biodiversity in protected and unprotected areas (Joshi and Samant, 2004). Therefore, present attempt has been made to prioritize the habitats and communities of the Lahaul valley in a proposed Cold Desert Biosphere Reserve. The habitat denotes the physical conditions that surround a species, or species population, or assemblage of species, or community (Clements and Shelford, 1939). Today, habitat destruction is a major factor in causing a species population to decrease, eventually leading to its being endangered, or even to its extinction. Due to sparse distribution of forest vegetation in Lahaul valley, the identified forest communities (14) were less than other Biosphere Reserves of the IHR (Samant et al., 2002). This may be due to the severe cold climatic conditions of the area. Mostly coniferous communities with wide range of distribution were recorded. Broad leaved communities were found in few patches and had narrow range of distribution. The loss of biodiversity is a growing concern throughout in the globe. The reasons are over exploitation and habitat destruction of the biodiversity elements. Therefore, protection of natural habitats is essential. Prioritization of habitats and communities supporting high species diversity and native, endemic, economically important and threatened species would help to some extent for the conservation of biodiversity.

Amongst the communities, Juniperus polycarpos, Pinus wallichiana, Cedrus deodara, Picea smithiana, Betula utilis and Abies pindrow showed high species richness, native, endemic, near-endemic, economically important and threatened species.

Significant positive correlations between species richness and native species $(\mathrm{r}=0.98, \mathrm{p}$ $<0.01, \mathrm{n}=14)$ (Figure 3a); species richness and endemic species $(\mathrm{r}=0.96, \mathrm{p}<0.01, \mathrm{n}=$ 14) (Figure 3b); and native and endemic species $(\mathrm{r}=0.97, \mathrm{p}<0.01, \mathrm{n}=14)$ within the identified communities were found (Figure $3 c$ ). Like other parts of the IHR, in Lahaul valley, the percentage of native and endemic species increased with the altitude and species richness decreased. Regular monitoring of the habitats and populations of the native and endemic species facing high anthropogenic pressure is essentially required, so that adequate planning for their conservation management could be done intime.

In the IHR, most of the studies related to prioritization of species for conservation have been carried out using qualitative attributes/observations, only. Assessment of status of the species for prioritization using qualitative as well as quantitative attributes has been suggested by few workers (Joshi and Samant, 2004; Samant et al., 1996b; 1998a; 2001). Further, assessment status and values of the communities for conservation is urgently required (Joshi and Samant, 2004). In the present study, amongst habitats, forests (44), shady moist (38), respectively, and amongst communities, Abies pindrow - Pinus wallichiana mixed, Fraxinus xanthoxyloides, Picea smithiana - Pinus wallichiana mixed, Cedrus deodara - Acer cappadocicum mixed, Hippophae salicifolia, Juglans regia - Ulmus wallichiana - Acer acuminatum mixed, Juniperus polycarpos - Cedrus deodara mixed, Betula utilis and Salix daphnoides, respectively showed the high CPI, hence prioritized for conservation. 

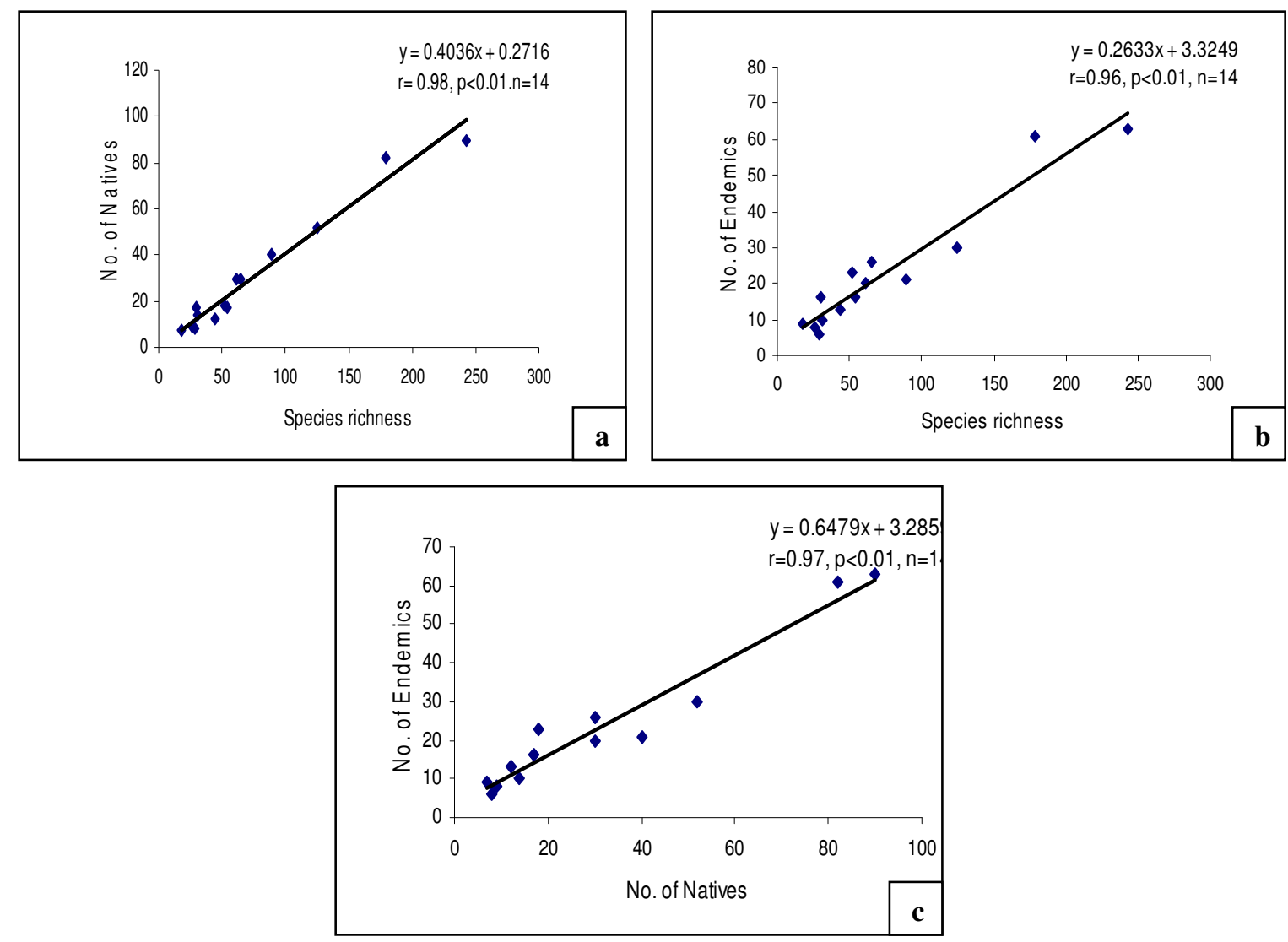

Figure 3. Correlations between a) Species richness and Naives species; b) Species richness and Endemic species; and c) Native and Endemic species in Lahaul valley of the proposed CDBR

These habitats and communities, requires regular monitoring, so that adequate management of these habitats and communities could be done intime. Some of the communities, such as Juniperus polycarpos, Pinus wallichiana, Cedrus deodara, Picea smithiana, Betula utilis and Abies pindrow showed wide range of distribution. However, typical topography, severe climatic conditions and high degree of anthropogenic pressure i.e., collection of fuel, fodder, timber, etc. and grazing by the sheeps of the nomadic shepherd (i.e., gaddies, gujjars and locals) have resulted in rapid loss of biodiversity elements of these communities. According to forest policy 1988 of India, the area under forest in hilly region should be $66 \%$ of its geographical area. The recorded area under actual forest cover during year 2003 was $180 \mathrm{~km}^{2}$ in Lahaul and Spiti district out of total $13,835 \mathrm{~km}^{2}$, which is too less (Gupta, 2007). Promotion of Afforestation programmes through plantation of native species may help in increasing the vegetation cover in the area.

\section{Conclusion}

The present study gives comprehensive information for the first time about the status of forests distributed under different habitats and communities in Lahaul valley a part of proposed CDBR. The recorded species richness under forest communities and habitats was 
relatively less to other Biosphere Reserves of the IHR, may be due to adverse climatic conditions prevailing in the area. The variation in microclimate of an area showed great variability in species richness among different communities, habitats and aspects. The communities and habitats with wide range of distribution represented high species richness, high number of native, endemic, economically important and threatened species. Most communities showed comparatively less regeneration, indicative of great threat to these forests in future. Also, applicability of sustained forest management principles right from the plantation upto harvesting is lacking. As the area under cold desert is about one fourth part of total geographical area in Himachal Pradesh, there is a need to increase area under plantation. The conservation of broad-leaved communities is important for improving soil fertility status and to maintain the ecosystem conducive for regeneration establishment. The native and introduced fast growing hardy species should be planted through social forestry and agroforestry schemes to revive the habitats. Plantation of native and endemic species like Abies pindrow, Fraxinus xanthoxyloides, Hippophae salicifolia, Pinus wallichiana, Cedrus deodara, Picea smithiana, Populus ciliata, Juglans regia, Corylus jacquemontii, Acer acuminatum etc., is important to increase the forest cover of the area. Hippophae salicifolia, the most demanded species in market for edible and medicinal values, showed high density with less basal area. The pure stand communities like Hippophae salicifolia and Juniperus polycarpos need much attention for protection against fire, diseases, high anthropogenic and abiotic pressures. The presence of moderately high species richness, native, endemic and threatened species enhances the conservation value of the area. The regular monitoring of communities and habitats with high CPI is essentially required for their proper management. The communities located near habitations showed high anthropogenic pressure than that of distant communities. The protective measures of key stone species against adverse climate should be encouraged for conservation. In a nutshell, it is suggested that proper strategy and policy dealing with conservation management for prioritized communities and habitats should be formulated so that effective management of forests could be achieved in posterity.

Acknowledgements. Authors thank to Dr. U. Dhar, Director of the Institute for providing necessary facilities. Thanks are also due to Dr. Manohar Lal for help during field study. Financial assistance received from Ministry of Environment and Forests New Delhi (Letter No.: 08/27/04-CS/BR; Dated- 27.06.2005), is greatly acknowledged.

\section{REFERENCES}

[1] Anonymous (1883-1970): Index Kewensis Plantarum Phanerogamarum Vol. 1-2 (1883-1885) and 15 Suppl. (1886-1970). - Clarendron Press, Oxford.

[2] Anonymous (2007): Biodiversity hot spots. http://www.biodiversityhotspots.org/xp/hotspots/himalaya/pages/default.apx.

[3] Arya, S.C. (2002): Assessment of habitat diversity, distribution of vegetation and human dependence in alpine meadows of Nanda Devi Biosphere Reserve, West Himalaya. Ph.D. Thesis submitted to Kumaun University, Nainital, India.

[4] Aswal, B.S., Mehrotra, B.N. (1994): Flora of Lahaul-Spiti (a cold desert in North-West Himalaya). - Bishen Singh and Mahendra Pal Singh, Dehradun. 
[5] Chowdhery, H.J., Wadhwa, B.M. (1984): Flora of Himachal Pradesh, Vols. 1-3. - Botanical Survey of India, Calcutta, India.

[6] Clements, F.E., Shelford, V.E. (1939): Bio-ecology. - John Wiley \& Sons, Inc., New York.

[7] Curtis, J.T., Mc Intosh (1950): The interrelation of certain analytic and phytosociological characters. - Ecology 31: 434-455.

[8] Dhaliwal, D.S., Sharma, M. (1999): Flora of Kullu District (Himachal Pradesh). - Bishen Singh Mahendra Pal Singh, Dehra Dun, India.

[9] Dhar, U., Rawal, R.S., Samant, S.S. (1997): Structural diversity and representative ness of forest vegetation in a protected area of Kumaun Himalaya, India: implications for conservation. - Biodiversity and Conservation 6: 1045-1062.

[10] Dhar, U., Rawal, R.S., Upreti, J. (2000): Setting priorities for conservation of medicinal plants: A case study in the Indian Himalaya. - Biological Conservation 95: 57-65.

[11] Dhar, U., Samant, S.S. (1993): Endemic diversity of Indian Himalaya I. Ranunculaceae and II. Paeoniaceae. - Journal of Biogeography 20: 659-668.

[12] Greig-Smith, P. (1957): Quantitative Plant Ecology. - Academic Press, New York.

[13] Gupta, K.G. (2007): Deforestation and forest cover changes in the Himachal Himalaya, India. - International Journal of Ecology and Environmental Science 33(2-3): 207-218.

[14] Joshi, H.C. (2002): Assessment of habitat diversity, forest vegetation and human dependence in the buffer zone of Nanda Devi Biosphere Reserve of West Himalaya. - Ph.D. Thesis submitted to Kumaun University Nainital, India.

[15] Joshi, H.C., Samant, S.S. (2004): Assessment of forest vegetation and prioritization of communities for conservation in a part of Nanda Devi Biosphere Reserve, West Himalaya, India I. - International Journal of Sustainable Development and World Ecology 11: 326-336.

[16] Kalakoti, B.S., Pangtey, Y.P.S., Saxena, A.K. (1986): Quantitative analysis of high altitude vegetation of Kumaun Himalaya. - Journal of Indian Botanical Society 65: 384-396.

[17] Maity, D., Chauhan, A.S. (2002): Assessment of plant diversity of Khangchendzonga Biosphere Researve in Sikkim. - In: Sharma, J.K., Easa, P.S., Mohanan, C., Sasidharan, N., Rai, R.K. (eds.) Biosphere Reserves in India and their Management, Kerala Forest Research Institute, Peechi, and Ministry of Environment and Forest, New Delhi, pp. 222-225.

[18] Misra, R. (1968): Ecological Work Book. - Oxford and IBH Publishing Company, Calcutta, India.

[19] Mueller-Dombois, D., Ellenberge, H. (1974): Aims and methods of vegetation ecology. John Willey and Sons, New York.

[20] Murti, S.K. (2001): Flora of cold deserts of western Himalaya. Vol. I. - Botanical survey of India, Calcutta, India.

[21] Nautiyal, B.P., Pandey, N., Bhatt, A.D. (1997): Analysis of vegetation pattern in alpine zone in north-west Himalaya: a case study of Garhwal Himalaya with special reference to diversity and distributional patterns. - International J. of Ecology and Environmental Sciences 23: 4965 .

[22] Pandey, S. (2006): Assessment of useful plants resources of the Gola Catchment of Kumaun. - Ph.D. Thesis submitted to Kumaun University Nainital.

[23] Pant, S., Samant, S.S. (2007): Assessment of plant diversity and prioritization of communities for conservation in Mornaula Reserve Forest. - Applied Ecology and Environmental Research 5(2): 151-166.

[24] Polunin, O., Stainton, A. (1984): Flowers of the Himalaya. - Oxford University Press, Oxford, India

[25] Rawal, R.S. Bankoti, N.S., Pangtey, Y.P.S. (1994): Broad community identification of high altitude forest vegetation in Pindari catchment of Kumaun. - Proceedings of Indian National Science Academy 60(6): 553-556.

APPLIED ECOLOGY AND ENVIRONMENTAL RESEARCH 8(2): 101-117. http://www.ecology.uni-corvinus.hu • ISSN 15891623 (Print) • ISSN 17850037 (Online) (c) 2010, ALÖKI Kft., Budapest, Hungary 
[26] Rawal, R.S., Pangtey, Y.P.S. (1994): Distribution and structural-functional attributes of trees in the high altitude zone of central Himalaya, India. - Vegetatio 112: 29-34.

[27] Rawat, G.S., Adhikari, B.S., Rana, B.S. (2001): Vegetation surveys in the Indian Trans Himalaya. - In: anonymous, (ed.) Conserving Biodiversity, in the Indian Trans Himalaya: New Initiatives of Field Conservation in Ladakh. Report, Wild life Institute of India, International Snow Leopard Trust, and U.S. Fish and Wildlife Service, pp. 7-14.

[28] Rawat, V.R.S., Kumar, P., Kumar, P. (1989): Ecological studies of some Cedrus deodara (deodar) forest in Western Himalayas, India. - Indian Journal of Forestry 12(2): 145-150.

[29] Rikhari, H.C., Chandra, R., Singh, S.P. (1989): Pattern of species distribution and community characters along a moisture gradient within an oak zone of Kumaun Himalaya. - Proceedings of Indian National Science Academy 55: 431-438.

[30] Samant, S.S., Butola, J.S., Sharma, A. (2007a): Assessment of diversity, distribution, conservation status and preparation of management plan for medicinal plants in the catchment area of Parvati hydroelectric project stage-III in northwestern Himalaya. - Journal of Mountain Science 4(1): 34-56.

[31] Samant, S.S., Dhar, U. (1997): Diversity, endemism and economic potential of wild edible plants of Indian Himalaya. - International Journal of Sustainable Development and World Ecology 4: 179-191.

[32] Samant, S.S., Dhar, U., Palni, L.M.S. (1998a): Medicinal plants of Indian Himalaya: diversity distribution potential values. - Gyanodaya Prakashan, Nainital, India.

[33] Samant, S.S., Dhar, U., Palni, L.M.S. (2001): Himalayan medicinal plants: potential and prospects. - Gyanodaya Prakashan, Nainital, India.

[34] Samant, S.S., Dhar, U., Rawal, R.S. (1996a): Natural resource use by some natives within Nanda Devi Biosphere Reserve in west Himalaya. - Ethnobotany 8: 40-50.

[35] Samant, S.S., Dhar, U., Rawal, R.S. (1996b): Conservation of rare endangered plants: The context of Nanda Devi Biosphere Reserve. - In: Ramakrishnan, P.S. Purohit, A.N. Saxena, K.G. Rao, K.S. and R.K. Maikhuri (eds.) Conservation and Management of Biological Resources in Himalaya.-Oxford and IBH Publishing Company Private Limited, New Delhi, India. pp. 521-545.

[36] Samant, S.S., Dhar, U., Rawal, R.S. (1998b): Biodiversity status of a protected area of west Himalaya. I-Askot Wildlife Sanctuary. - International Journal of Sustainable Development and World Ecology 5: 194-203.

[37] Samant, S.S., Joshi, H.C. (2004): Floristic diversity, community pattern and changes of vegetation in Nanda Devi National Park. - In: Biodiversity Monitoring Expedition, Nanda Devi 2003 (18 June to 8 July, 2003), Uttaranchal Forest Department Dehradun. pp. 39-54.

[38] Samant, S.S., Joshi, H.C., Arya, S.C., Pant, S. (2002): Studies on the structure, composition and changes of the vegetation in Nanda Devi Biosphere Reserve of wWest Himalaya - Final Technical Report Submitted to Ministry of Environment and Forests, New Delhi, India.

[39] Samant, S.S., Pal, M. (2003): Diversity and conservation status of medicinal plants in Uttaranchal State. - Indian Forester 129(9): 1090-1108.

[40] Samant, S.S., Joshi, H.C., Arya, S.C., Pant, S. (2003): Planning for the conservation and management of natural resources using participatory approach: A case study from Pindari area of Nanda Devi Biosphere Reserve, West Himalaya, India. - Van Vigyan 38(1-4): 41-54.

[41] Samant, S.S., Pant, S., Singh, M., Lal, M., Singh, A., Sharma, A., Bhandari, S. (2007b): Diversity, distribution pattern, indigenous uses and conservation prioritization of medicinal plants of Himachal Pradesh, India. - International Journal of Biodiversity Science and Management 3: 234-251.

APPLIED ECOLOGY AND ENVIRONMENTAL RESEARCH 8(2): 101-117. http://www.ecology.uni-corvinus.hu • ISSN 15891623 (Print) • ISSN 17850037 (Online) (c) 2010, ALÖKI Kft., Budapest, Hungary 
[42] Samant, S.S., Singh, M., Lal, M., Pant, S. (2007c): Diversity, Distribution and Prioritization of Fodder Species for Conservation in Kullu District, North-Western Himalaya, India. Journal of Mountain Science 4(3): 259-274.

[43] Saxena, A.K., Singh, J.S. (1982): A phytosociological analysis of woody species in forest communities of a part of Kumaun Himalaya. - Vegetatio 50: 3-22.

[44] Singh, D.K., Hajra, P.K. (1996): Floristic diversity. - In: Gujral, G.S. and V. Sharma (eds.) Biodiversity status in the Himalaya. British Council, New Delhi, India. pp. 23-38.

[45] Singh, J.S., Singh, S.P. (1992): Forest of Himalaya: Structure, Functioning and Impact of Man. - Gyanodaya Prakashan, Nainital.

[46] Singh, S.K., Rawat, G.S. (1999): Floral diversity and vegetation structure in Great Himalayan National Park, Western Himalaya. - Final Technical Report, FREEP-GHNP research project, Wild life Institute of India Dehradun.

[47] Singh, S.P., Adhikari, B.S., Garkoti, S.C., Rawat, Y.S. (1996): Structural and functional characteristics of the forest ecosystems around NDBR. - In: Ramkrishan, P.S. Purohit, A.N. Saxena, K.G. Rao, K.S. and R.K. Maikhuri (eds.) Conservation and Management of Biological Resources in Himalaya. - Oxford and IBH Publication Company Private Limited, New Delhi, India. pp. 521-545.

[48] Sinha, S.K., Samant, S.S. (2006): Climate change in the higher Himalayas - a case study in Lahaul Valley. - ENVIS News letter: Himalayan Ecology 3: 3-4. 